EXTENDED REPORT

\title{
Retinal blood flow measurements and neuroretinal rim damage in glaucoma
}

\author{
J F J Logan, S J A Rankin, A J Jackson
}

Br J Ophthalmol 2004;88:1049-1054. doi: 10.1136/bjo.2003.034884

See end of article for authors' affiliations .....................

Correspondence to: Dr J Logan, Department of Ophthalmology, The Royal Group of Hospitals, Grosvenor Road, Belfast BT1 2 6BA, Northern Ireland, UK; joanne. logan1@btopenworld.com

Accepted for publication 15 December 2003

\begin{abstract}
Aim: To assess retinal blood flow characteristics in subjects with normal tension glaucoma (NTG), primary open angle glaucoma (POAG), and a group of controls using the Heidelberg retina flowmeter (HRF). The vascular parameters were correlated against structural damage of the optic nerve head, assessed using the Heidelberg retina tomograph (HRT).

Methods: HRF images were obtained in 76 subjects with NTG, 58 with POAG, and 38 controls. Optic nerve head images, acquired using the HRT, were analysed with Moorfields Regression Analysis software. The HRF variables, measured adjacent to a rim segment identified as "abnormal," were compared with the vascular parameters of the "normal" rim segments. The HRF parameters of the segments identified as normal in glaucoma subjects were compared with matched control segments.

Results: The glaucoma subjects had significantly lower retinal haemodynamics than the control subjects. There were no significant differences in the HRF parameters between the NTG and POAG subjects. The discs that had been identified as having abnormal segments had lower HRF values than those with a corresponding normal segment. The glaucoma subjects with normal rim segments had statistically significant lower velocity, flow, and volume measurements than the controls for each location sampled. Conclusion: This study shows a relation between structural damage of the optic nerve head and the level of retinal blood flow. The changes in the circulation could indicate that it may be an early marker of the pathological process.
\end{abstract}

$\mathrm{T}$ he vascular aetiology of glaucoma hypothesises that a compromised blood supply to the optic nerve head contributes to optic nerve head damage. Localised damage may occur when the ocular perfusion pressure falls outside the normal range of autoregulation. This may be the result of a systemic dysfunction (low systemic blood pressure, large nocturnal dips in blood pressure, or peripheral vasospastic disorders $),{ }^{1-5}$ or a local abnormality in the ocular blood supply. ${ }^{6-8}$

The Heidelberg retina flowmeter (HRF, Heidelberg Engineering GmbH, Heidelberg, Germany) allows noninvasive quantification of the perfusion of the retina and optic nerve head. ${ }^{9}$ The use of a confocal laser in this instrument has refined the technique of laser Doppler flowmetry by allowing discrete areas of the retina to be examined with an axial resolution of $300 \mu \mathrm{m} .{ }^{9}$ The incorporation of a scanning technique allows multiple sampling measurements over the area of interest. These measurements are utilised in the fast Fourier transformation algorithm. The validity and reproducibility of the technique have been well documented..$^{9-12}$

The aim of this study was to compare the blood flow, volume and velocity as measured by the HRF between subjects with normal tension glaucoma (NTG), primary open angle glaucoma (POAG) and a group of controls. These vascular parameters were correlated against structural damage on the optic nerve head, as assessed using the Heidelberg retina tomograph (HRT, Heidelberg Engineering $\mathrm{GmbH}$, Heidelberg, Germany).

\section{PATIENTS AND METHODS}

In all, 134 subjects with glaucoma were recruited from a specialist glaucoma clinic. The inclusion criteria for subjects were: glaucomatous optic nerve damage in at least one eye (this included a localised notch, diffuse erosion of the neuroretinal rim, shallow saucerised cupping, enlargement of the cup, or a retinal nerve fibre layer defect), bilateral open anterior chamber angles, and a reproducible Humphrey visual field defect with the 24-2 full threshold strategy as defined by Anderson. ${ }^{13}$ This was confirmed on at least two previous field plots. Unreliable visual fields plots were excluded if the false positives, false negatives, or fixation losses exceeded $30 \%$. Subjects with POAG had to have an untreated intraocular pressure (IOP) of more than $21 \mathrm{~mm} \mathrm{Hg}$ in at least one eye. NTG subjects had to have a median IOP of less than $21 \mathrm{~mm} \mathrm{Hg}$ on a diurnal curve of no less than five readings, in both eyes. One spike of up to $24 \mathrm{~mm} \mathrm{Hg}$ was accepted. Subjects on topical medications underwent a 2 week washout period before testing.

Thirty nine control subjects volunteered for the study. These individuals were either the spouses of the glaucoma subjects, or were alternatively recruited from private optometric practice. The inclusion criteria for the controls were no history of glaucoma in either eye, bilateral healthy optic nerve heads, a Humphrey visual field 24-2 full threshold strategy showing no evidence of glaucomatous defects, and an IOP of less than $21 \mathrm{~mm} \mathrm{Hg}$. Lens opacities in one of the control subjects caused a reduction in the mean deviation, the range in the entire group being $-5.88 \mathrm{~dB}$ to $1.76 \mathrm{~dB}$. This subject had poor image quality owing to a combination of small pupils and lens opacities. The images were subsequently excluded from analysis.

All subjects had a spherical refractive error of less than 7 dioptres and astigmatism of less than 2 dioptres. Corrected visual acuities of better than $\log$ MAR 0.3 (6/12) were also required. One eye of each subject was included in the analysis. The eye with the most advanced glaucomatous damage was chosen for the glaucoma subjects, and chosen

Abbreviations: HRF, Heidelberg retina flowmeter; NTG, normal tension glaucoma; PNR, perfusion/nerve fibre ratio; POAG, primary open angle glaucoma 

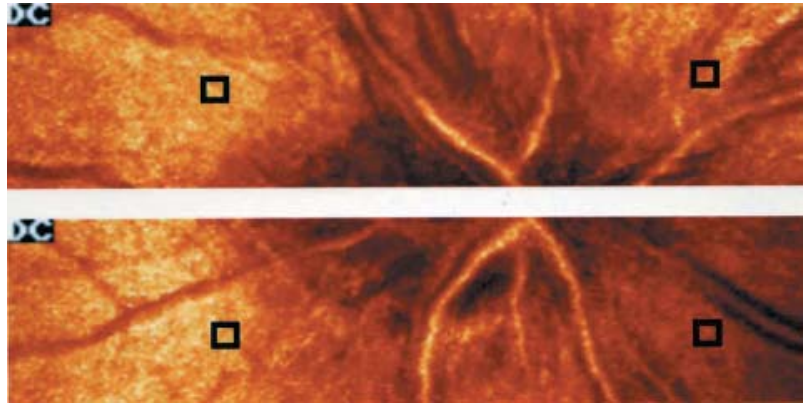

Figure 1 HRF images showing the four locations sampled using the $10 \times 10$ pixel box.

randomly for the controls. All subjects were requested to refrain from smoking and consuming caffeine on the day of the study.

Ethical approval was obtained from the research ethics committee, Faculty of Medicine, Queen's University Belfast. Informed written consent was obtained from each subject. The research adhered to the tenets of the Declaration of Helsinki.

A total of six HRF images were obtained for each eye; three in the region of the superior optic nerve head and adjacent retina and three in the region of the inferior optic nerve head and adjacent retina. Images were focused on the superficial retina. The patients were requested to keep their chin on the chin rest until all the images were acquired. A Fourier transformation generated a two dimensional perfusion map of each image. The three images for each location were assessed and the best, in terms of clarity and illumination, was used for further analysis. The perfusion maps were used to determine blood flow, volume, and velocity in arbitrary units using the $10 \times 10$ pixel box at $200 \mu \mathrm{m}$ from the disc margin. Care was taken to ensure blood vessels and saccades were avoided when positioning the box. The locations measured were; superior-temporal retina, superior-nasal retina, inferior-temporal retina, and inferior-nasal retina (fig 1). Images recorded during excessive eye movement were rejected.

The Heidelberg retina tomograph (HRT), software version 2.01, was used to acquire three optic nerve images with a field of $10^{\circ} \times 10^{\circ}$. Keratometry values were entered for each eye to correct for magnification errors. The mean topography image was calculated and the optic disc margin was drawn at the inner edge of the scleral ring. Images were analysed using Moorfields Regression Analysis software on a peripheral PC. A normal range for this software has been defined by the $99 \%$ prediction interval of the linear regression of log neuroretinal rim area against optic disc area, derived from a group of normals. ${ }^{14}$ When the global rim area or one of the predefined segments of the neuroretinal rim falls outside this normal range it is labelled as "abnormal." There are six predefined segments; nasal, nasal-inferior, nasal-superior, temporal, temporal-inferior, and temporal-superior. The HRF variables measured adjacent to a rim segment identified as abnormal were compared with the vascular parameters of the "normal" rim segments. For example, if the Moorfields software identified the disc as having an abnormal inferior-temporal segment, the inferior-temporal HRF parameters would be compared against those glaucomatous discs with a normal inferior-temporal segment (fig 2). The HRF parameters of the segments identified as normal in glaucoma subjects were compared against the controls.

The values for HRF volume, flow, and velocity and the quantitative measurements for the optic nerve head parameters were exported to an Excel spreadsheet. The data were checked using histograms to ensure they was approximately normally distributed. The data were analysed using the Student's $t$ test for two groups and one way analysis ANOVA if there were more than two groups. $\chi^{2}$ tests were used to compare categorical data between groups. A significant value was taken to be $\mathrm{p}<0.05$. SPSS for Windows software, version 10 , was used to perform the statistical analysis.

\section{RESULTS}

Seventy six subjects with NTG, 58 subjects with POAG, and 38 controls took part in this study. The subjects with POAG have a significantly higher IOP than the subjects with NTG and the controls. The widespread of IOP measured in the POAG subjects on the day of the research session represents at one extreme, those that have low a IOP post-trabeculectomy, ranging to high untreated levels following washout of topical medication. Twenty eight per cent $(n=37)$ of glaucoma subjects and 15\% $(n=6)$ of controls had to be excluded from the HRF analysis because of poor image quality arising from problems with fixation, eye movement, or lens opacities. There were 97 eyes of glaucoma subjects and 33 control eyes included in the final analysis. The characteristics of the study population can be seen in table 1 .

The HRF parameters for the glaucoma and control subjects are outlined in table 2. The glaucoma subjects have a significantly lower retinal blood volume, flow, and velocity than the control subjects at the two superior and inferior retinal locations $(\mathrm{p}<0.001)$.

When the NTG and POAG subjects are compared, the inferior-temporal volume values are slightly lower for the NTG subjects $(p=0.025)$. This value loses significance when the large numbers of comparisons are taken into account. There are no significant differences between the remaining the HRF parameters (table 3 ).
A

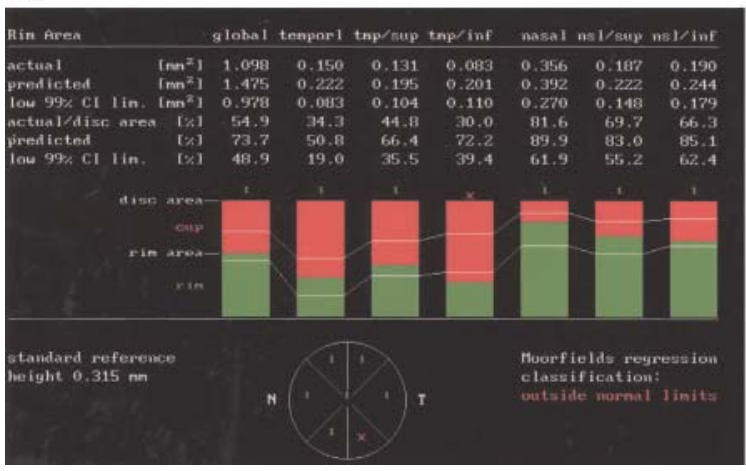

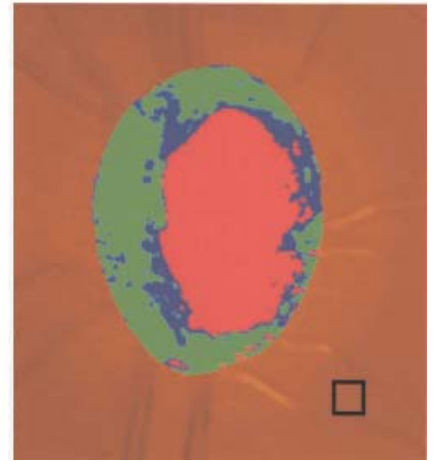

Figure 2 The temporal-inferior neuroretinal rim segment is classified abnormal by Moorfields regression analysis in this subject (A). The HRF parameters are measured adjacent to the "abnormal" segment using the $10 \times 10$ pixel box $(B)$ and are compared to the temporal-inferior HRF parameters of glaucomatous discs with a temporalinferior segment "within normal limits." 
Table 1 Characteristics of the study population (mean (SD))

\begin{tabular}{|c|c|c|c|c|}
\hline & NTG $(n=56)$ & POAG $(n=41)$ & Controls $(n=33$ ) & p Value \\
\hline Age (years) & $65.8(10.3)$ & $65.3(8.9)$ & $61.2(11.2)$ & $0.11^{*}$ \\
\hline Male:female (ratio) & $36: 64$ & $56: 44$ & $44: 56$ & $0.14 \dagger$ \\
\hline Right:left eyes (ratio) & $52: 48$ & $46: 54$ & $56: 44$ & $0.70+$ \\
\hline Refraction (D) & $+0.41(2.01)$ & $+0.45(1.73)$ & $+0.57(2.35)$ & $0.96^{*}$ \\
\hline Peak IOP (mm Hg) (range) & $16.32(2.64)(10-22)$ & $29.76(5.39)(23-50)$ & $14.75(3.03)(6-20)$ & $<0.001^{*}$ \\
\hline Test IOP (mm Hg) (range) & $17.11(2.91)(10-23)$ & $24.54(6.68)(9-38)$ & $14.75(3.03)(6-20)$ & $<0.001^{*}$ \\
\hline Visual field MD (dB) (range) & $-10.39(6.47)(-27.5$ to -0.7$)$ & $-9.42(4.86)(-21.2$ to -2.6$)$ & $-0.78(1.32)(-3.8$ to 1.8$)$ & $<0.001^{*}$ \\
\hline
\end{tabular}

To give an overall impression of the retinal blood flow the measurements of the four retinal locations (superior-nasal, superior-temporal, inferior-nasal, and inferior temporal) were averaged to give a value for "total volume," "total flow," and "total velocity." Again the measurements for the glaucoma subjects were significantly lower than the controls $(\mathrm{p}<0.001)$. There was no significant difference observed between the two glaucoma groups. The spread of the total volume, flow and velocity for each group is displayed in figure 3.

The HRF parameters in the optic discs with an abnormal superior-nasal, superior-temporal, inferior-nasal and inferior-temporal rim segment were compared against the glaucoma subjects with a normal segment. The discs that have been identified as having an abnormal inferior-temporal segment have statistically lower values for volume (14.40 (4.19) and 16.16 (3.51), $\mathrm{p}=0.045)$ and flow (218.28 (69.26) and $247.22(60.21), p=0.049)$ than the discs with a corresponding normal segment. The remaining HRF parameters for the abnormal segments show a trend towards lower values, although significance is not achieved (table 4).
The glaucoma subjects with normal rim segments have statistically significant lower velocity, flow, and volume measurements than the controls for each location sampled. For example at the inferior-temporal measurement location the glaucomatous subjects with a normal segment have a blood flow of $247.22(60.21)$ and the blood flow in the controls is $313.22(60.21)(\mathrm{p}<0.001)$ (fig 4$)$.

\section{DISCUSSION}

This study confirms previous findings of a reduced blood flow in the peripapillary retina of glaucomatous eyes while using the HRF. ${ }^{75}$ The four peripapillary retinal locations sampled (superior-nasal, superior-temporal, inferior-nasal, and inferior-temporal) all had significantly reduced levels of blood volume, flow, and velocity in the glaucoma subjects. This decrease in blood flow parameters at the four peripapillary retinal locations has not been observed in all studies. Nicolela et al found reduced levels in a temporal site to the optic nerve head just above the horizontal midline, but no significant differences between the glaucoma patients and controls in superior and inferior temporal measurement sites. ${ }^{7}$

Table 2 HRF parameters for the glaucoma and control subjects (in arbitrary units)

\begin{tabular}{llll}
\hline HRF parameter (mean (SD) & Glaucoma subjects $(\mathbf{n}=\mathbf{9 7})$ & Controls $(\mathbf{n}=\mathbf{3 3})$ & $\mathbf{p}$ Value \\
\hline Superior-nasal volume & $13.98(3.51)$ & $16.98(4.77)$ & $<0.001$ \\
Superior-nasal flow & $226.02(81.55)$ & $301.36(85.36)$ & $<0.001$ \\
Superior-nasal velocity & $0.80(0.29)$ & $1.06(0.29)$ & $<0.001$ \\
Superior-temporal volume & $15.32(3.95)$ & $18.25(3.94)$ & $<0.001$ \\
Superior-temporal flow & $237.16(70.63)$ & $291.80(72.42)$ & $<0.001$ \\
Superior-temporal velocity & $0.84(0.24)$ & $1.03(0.25)$ & $<0.001$ \\
Inferior-nasal volume & $13.38(3.67)$ & $15.63(4.03)$ & $<0.001$ \\
Inferior-nasal flow & $222.44(76.53)$ & $238.66(87.84)$ & $<0.001$ \\
Inferior-nasal velocity & $0.79(0.26)$ & $1.02(0.29)$ & $<0.001$ \\
Inferior-temporal volume & $14.97(4.05)$ & $18.33(3.64)$ & 0.004 \\
Inferior-temporal flow & $227.53(67.56)$ & $313.67(66.70)$ & $<0.001$ \\
Inferior-temporal velocity & $0.81(0.23)$ & $1.11(0.23)$ & $<0.001$ \\
\hline
\end{tabular}

Table 3 HRF parameters for the NTG and POAG subjects (in arbitrary units)

\begin{tabular}{llll}
\hline HRF parameters (mean (SD) & NTG $(\mathbf{n}=\mathbf{5 6})$ & POAG $(\mathbf{n}=\mathbf{4 1})$ & p Value \\
\hline Superior-nasal volume & $14.11(3.67)$ & $13.80(3.29)$ & 0.673 \\
Superior-nasal flow & $216.22(79.23)$ & $239.64(83.73)$ & 0.162 \\
Superior-nasal velocity & $0.77(0.28)$ & $0.86(0.29)$ & 0.130 \\
Superior-temporal volume & $15.49(4.53)$ & $15.08(3.02)$ & 0.614 \\
Superior-temporal flow & $230.20(70.33)$ & $246.83(70.77)$ & 0.252 \\
Superior-temporal velocity & $0.82(0.24)$ & $0.88(0.25)$ & 0.209 \\
Inferior-nasal volume & $14.09(4.04)$ & $12.41(2.86)$ & 0.835 \\
Inferior-nasal flow & $228.94(82.61)$ & $213.57(67.33)$ & 0.206 \\
Inferior-nasal velocity & $0.82(0.28)$ & $0.77(0.24)$ & 0.119 \\
Inferior-temporal volume & $14.89(4.50)$ & $15.07(3.39)$ & 0.025 \\
Inferior-temporal flow & $220.09(72.99)$ & $237.70(58.71)$ & 0.311 \\
Inferior-temporal velocity & $0.78(0.25)$ & $0.85(0.21)$ & 0.364 \\
\hline
\end{tabular}



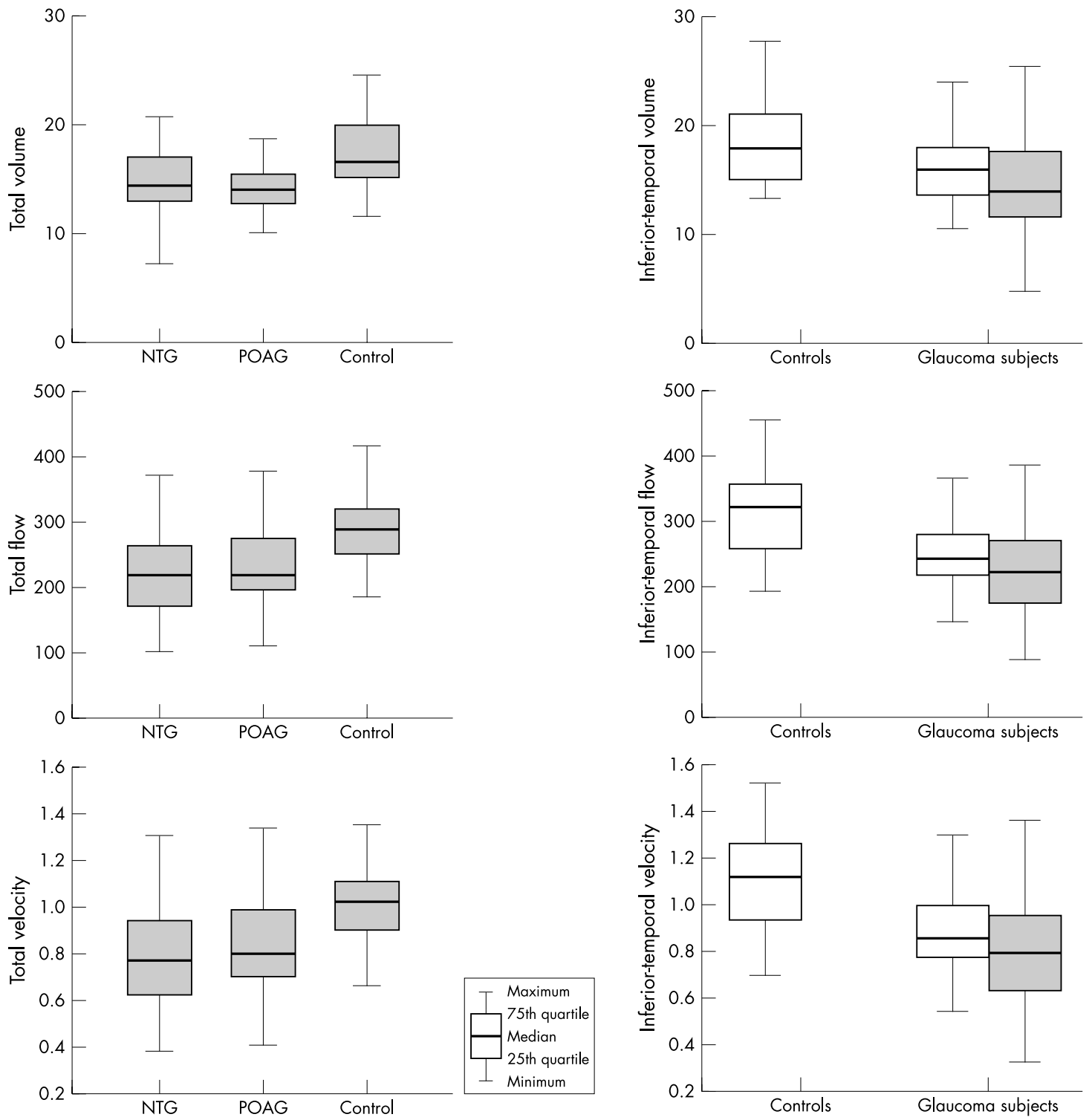

Figure 3 The spread of total volume, flow and velocity measurements in the NTG, POAG, and control subjects (averaging the data from the four quadrants).

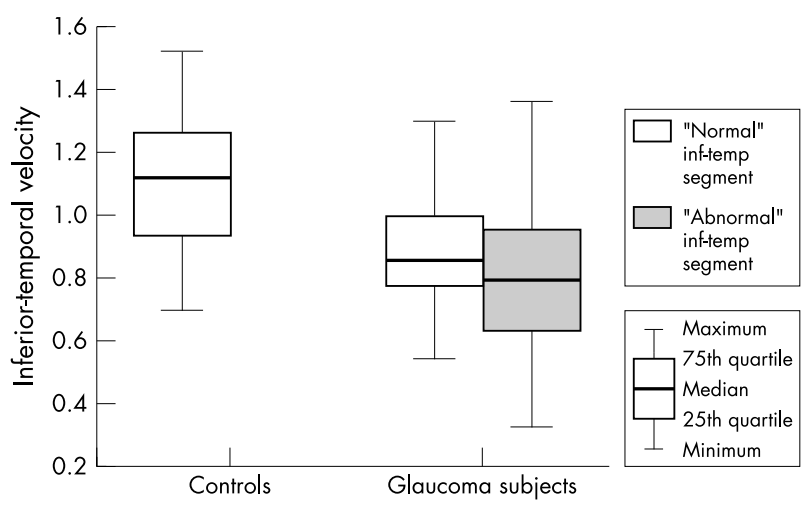

Figure 4 The spread of inferior-temporal volume, flow, and velocity measurements in the glaucoma subjects with "abnormal" and "normal" inferior-temporal rim segments, and the control subjects.

Table 4 HRF parameters in the "abnormal" and "normal" disc segments of the glaucoma subjects and the controls

\begin{tabular}{|c|c|c|c|}
\hline HRF parameter (mean (SD) & "Abnormal" disc segment & "Normal" disc segment & Controls \\
\hline Superior-nasal & $\mathrm{n}=45$ & $\mathrm{n}=52$ & $n=33$ \\
\hline volume & $13.30(3.56)$ & $14.56(3.39)$ & $16.98(4.77) \dagger$ \\
\hline flow & $210.58(89.40)$ & $239.12(72.53)$ & $301.36(85.36) \dagger$ \\
\hline velocity & $0.75(0.31)$ & $0.85(0.26)$ & $1.06(0.29) \dagger$ \\
\hline Superior-temporal & $n=44$ & $n=53$ & $n=33$ \\
\hline volume & $14.51(4.03)$ & $15.97(3.81)$ & 18.17 (3.97) $\ddagger$ \\
\hline flow & $235.07(70.94)$ & $238.86(70.99)$ & $291.00(73.43) \dagger$ \\
\hline velocity & $0.84(0.25)$ & $0.85(0.25)$ & $1.03(0.25) \dagger$ \\
\hline Inferior-nasal & $n=60$ & $n=37$ & $n=33$ \\
\hline volume & $13.90(4.01)$ & $12.54(2.88)$ & $15.63(4.10) \dagger$ \\
\hline flow & $227.32(78.18)$ & $214.52(74.14)$ & $285.16(87.95) \dagger$ \\
\hline velocity & $0.81(0.27)$ & $0.77(0.26)$ & $1.01(0.29) \dagger$ \\
\hline Inferior-temporal & $n=66$ & $n=31$ & $n=33$ \\
\hline volume & $14.40(4.19)$ & $16.16(3.51) \S$ & 18.33 (3.64)‡ \\
\hline flow & $218.28(69.26)$ & $247.22(60.21) \S$ & $313.22(60.21)^{*}$ \\
\hline velocity & $0.78(0.24)$ & $0.88(0.21)$ & $1.11(0.23)^{*}$ \\
\hline
\end{tabular}


There were no differences in the HRF measurements between the NTG and POAG subjects, although the POAG group had a higher intraocular pressure. Nicolela et al also found no difference between NTG and POAG subjects, although the study numbers were small ( 16 and 17 patients respectively). ${ }^{7}$

While the mean value for each of the HRF parameters are significantly lower in the NTG and POAG groups than the control group, a large degree of overlap exists. This spread of results limits the application of the HRF as a screening instrument.

This study shows a relation between structural damage of the optic nerve head as measured by the HRT, and the level of retinal blood flow. The inferior-temporal rim segments that have been identified as abnormal, by the Moorfields software in the HRT, have a significantly lower inferior-temporal blood flow than the glaucoma subjects with segments that have a normal rim. It has been shown that glaucomatous damage preferentially affects the inferior-temporal quadrant. ${ }^{16}$ This suggests that in early glaucomatous damage, when one segment of the optic nerve head is preferentially affected, the local retinal blood flow in this area may be reduced at this location.

The glaucomatous subjects classified as having a normal rim segment, by Moorfields regression software, have a significantly reduced peripapillary blood flow in the corresponding retinal location than the control group. This is similar to the results of a colour Doppler study which found that in subjects with unilateral glaucoma, the eye with a normal visual field and healthy optic disc had significantly lower blood flow velocity and higher resistance in their retrobulbar vessels, than the controls. ${ }^{17}$ This suggests that differences in the retinal haemodynamics may be able to be detected at an earlier stage than morphological damage. The changes in the circulation may indicate that it is an early marker of the pathological process, or that it may be a contributory factor in the pathogenesis of the disease process. The principle behind laser Doppler flowmetry should also be borne in mind. The combination of internal noise and the variations in intensity of back scattered light which are not caused by Doppler shifts is called "zero offset."11 18 This value means that even when the flow and velocity are zero, the measure obtained by HRF will always be greater than zero. Zero offset varies between individuals depending on the ocular media and the perfused tissue. It is conceivable that optic nerve structural damage may cause changes in the scattering of light and alter HRF measurements due to the internal noise signal.

A recent study evaluated the relation between retinal capillary blood flow as measured by the HRF and the morphology of the optic disc measured by HRT in a group of normals. ${ }^{19}$ A perfusion/nerve fibre ratio (PNR) was calculated to determine perfusion per unit of retinal nerve fibre layer. They found a significantly lower ratio in the inferior sector of the optic disc compared to the superior sector. The authors hypothesised that the inferior region of the optic disc may be more vulnerable to glaucomatous damage as a result of a lower blood flow per retinal nerve fibre layer.

Approximately $25 \%$ of the subjects had to be excluded from the HRF analysis because of poor quality images. This was mainly a result of eye movement causing saccades in the images, or image degradation because of lens opacities. The proportion of glaucoma subjects excluded is higher than the controls (28\% of glaucoma cases and $15 \%$ of controls). This may be because the control subjects have less lens opacities and better fixation than the glaucoma cases. Previous investigators have found similar problems with poor quality images, with $50-64 \%$ of glaucoma and $37 \%$ of control images being excluded. ${ }^{720}$
A limitation of this study was the sampling technique used to measure the blood flow. The $10 \times 10$ pixel box represents 100 different flow measurements. A slight shift of location may lead to a very different result. Every attempt was made to place the box at the same location inter-individually. Owing to variations in the retinal vasculature, the box may have been shifted slightly to avoid the blood vessels. More recent advances in the software have included an automatic full field perfusion analysis (AFFPIA). ${ }^{21}$ This analysis calculates the Doppler shift at each individual pixel. Saccades, blood vessels, and overexposed and underexposed pixels are excluded from the analysis. High values for intraobserver and interobserver reproducibility have been obtained..$^{21}{ }^{22}$ Other investigators have advocated using every pixel in the entire image for analysis, and to combine HRT and HRF to ensure a proper sensitivity for the signal from the neuroretinal rim. ${ }^{23}{ }^{24}$

The relation between the level of optic nerve head damage quantified structurally and a reduced retinal blood flow may prove useful in the understanding of the pathological process. This study does not tell us if the impaired circulation is a causative factor in the disease process, or if it is secondary as a result of the loss of axons in the optic nerve head. The reduced retinal circulation in glaucoma subjects identified as having a neuroretinal rim within normal limits at that location, suggests that it may be a causative factor in the pathogenesis of the disease, or an early marker of the pathological process.

\section{ACKNOWLEDGEMENTS}

This research was funded by grants from the Research and Development Office for the HPSS in Northern Ireland and the British Council for the Prevention of Blindness.

\section{Authors' affiliations}

J F J Logan, S J A Rankin, A J Jackson, Department of Ophthalmology, The Royal Group of Hospitals, Belfast, UK

J F J Logan, A J Jackson, Department of Ophthalmology, Queen's University Belfast, Belfast, UK

A J Jackson, Faculty of Biomedical Sciences, University of Ulster, Coleraine, Nothern Ireland, UK

\section{REFERENCES}

1 Hayreh SS, Zimmerman MB, Podhajsky $P$, et al. Nocturnal arterial hypotension and its role in optic nerve head and ocular ischemic disorders. Am J Ophthalmol 1994; 117:603-24.

2 Hayreh SS, Podhajsky P, Zimmerman MB. Role of nocturnal arterial hypotension in optic nerve head ischemic disorders. Ophthalmologica 1999;213:76-96.

3 Graham SL, Drance SM, Wijsman K, et al. Ambulatory blood pressure monitoring in glaucoma. The nocturnal dip. Ophthalmology 1995;102:61-9.

4 Phelps CD, Corbett JJ. Migraine and low tension glaucoma. A case control study. Invest Ophthalmol Vis Sci 1985;26:1105-8.

5 Drance SM, Douglas GR, Wiisman K, et al. Response of blood flow to warm and cold in normal and low-tension glaucoma patients. Am J Ophthalmol 1988;105:35-9.

6 Rankin SJA. Color Doppler imaging of the retrobulbar circulation in glaucoma. Surv Ophthalmol 1999;43(Suppl 1):S176-82.

7 Nicolela MT, Hnik P, Drance SM. Scanning laser Doppler flowmeter study of retinal and optic disk blood flow in glaucomatous patients. Am J Ophthalmol 1996;122:775-83.

8 Trew DR, Smith SE. Postural studies in pulsatile ocular blood flow: II Chronic open angle glaucoma. Br J Ophthalmol 1991;75:71-75.

9 Michelson G, Schmauss B. Two dimensional mapping of the perfusion of the retina and optic nerve head. Br J Ophthalmol 1995;79:1126-32.

10 Michelson G, Schmauss B, Langhans MJ, et al. Principle, validity, and reliability of scanning laser Doppler flowmetry. J Glaucoma 1996;5:99-105.

11 Chauhan BC, Smith FM. Confocal scanning laser Doppler flowmetry: experiments in a model flow system. J Glaucoma 1997;6:237-45.

12 Nicolela MT, Hnik P, Schulzer M, et al. Reproducibility of retinal and optic nerve head blood flow measurements with scanning laser Doppler flowmetry. J Glaucoma 1997;6:157-64.

13 Anderson DR. Automated static perimetry. St Louis: Mosby Year Book, 1992.

14 Wollstein G, Garway-Heath DF, Hitchings RA. Identification of early glaucoma cases with the scanning laser ophthalmoscope. Ophthalmology 1998; 105:1557-63. 
15 Michelson G, Groh M, Langhans M. Perfusion of the juxtapapillary retina and neuroretinal rim area in primary open angle glaucoma. J Glaucoma 1996;5:91-8.

16 Jonas JB, Fernandez MC, Sturmer J. Pattern of glaucomatous neuroretinal rim loss. Ophthalmology 1993;100:63-8.

17 Nicolela MT, Drance SM, Rankin SJA, et al. Color Doppler imaging in patients with asymmetric glaucoma and unilateral visual field loss. Am J Ophthalmo 1996; 121:502-10.

18 Tsang AC, Harris A, Kagemann L, et al. Brightness alters Heidelberg retinal flowmeter measurements in an in vitro model. Invest Ophthalmol Vis Sci 1999:40:795-9.

19 Harris A, Ishii Y, Chung HS, et al. Blood flow per unit retinal nerve fibre tissue volume is lower in the human inferior retina. $\mathrm{Br} J$ Ophthalmol 2003:87:184-8.
20 Kerr J, Nelson P, O'Brien C. A comparison of ocular blood flow in untreated primary open-angle glaucoma and ocular hypertension. Am J Ophthalmol 1998; 126:42-51

21 Michelson G, Welzenbach J, Pal I, et al. Automatic full field analysis of perfusion images gained by scanning laser Doppler flowmetry. Br J Ophthalmol 1998:82:1294-300.

22 lester M, Altieri M, Michelson G, et al. Intraobserver reproducibility of a twodimensional mapping of optic nerve head perfusion. J Glaucoma 2002;11:488-92

23 Chung HS, Harris A, Kagemann L, et al. Peripapillary retinal blood flow in normal tension glaucoma. Br J Ophthalmol 1999;83:466-9.

24 Jonescu-Cuypers CP, Chung HS, Kagemann L, et al. New neuroretinal rim blood flow evaluation method combining Heidelberg retina flowmetry and tomography. Br J Ophthalmol 2001;85:304-9. 